\title{
Cochrane continues to seek stable funding
}

A ppeals from Cochrane Canada and its supporters to the Canadian Institutes of Health Research (CIHR) and the Federal Health Minister Jane Philpott to restore its direct funding have not been met. Yet, Cochrane executives — including new director Dr. Holger Schünemann — remain optimistic.

An online petition by Evidence for Democracy asking Philpott to restore federal funding garnered more than 2000 signatures since January.

Cochrane Canada's \$2-million annual primary funding from CIHR through a peer-reviewed, but noncompetitive process, ended in 2015. Instead, the federal agency said it will fund Cochrane through competitive grant programs.

The directed grant approach is no longer appropriate because Cochrane isn't the only group producing systematic reviews in Canada, according to an Apr. 27 statement from Philpott's office. It said Cochrane has been encouraged to apply for other CIHR funding.

A virtually identical statement from CIHR on May 2 also noted that it "continues to engage with Cochrane Canada to help identify funding opportunities."

Although other individuals are doing systematic reviews in Canada, they are doing them more expensively and without the added value that Cochrane provides, said Dr. Jeremy Grimshaw, who was director of Cochrane Canada for 11 years. He estimates that the typical CIHR knowledge synthesis grant is $\$ 100000$ - five times the cost of a Cochrane review. In addition to producing reviews, Cochrane also trains new researchers in its methodology.

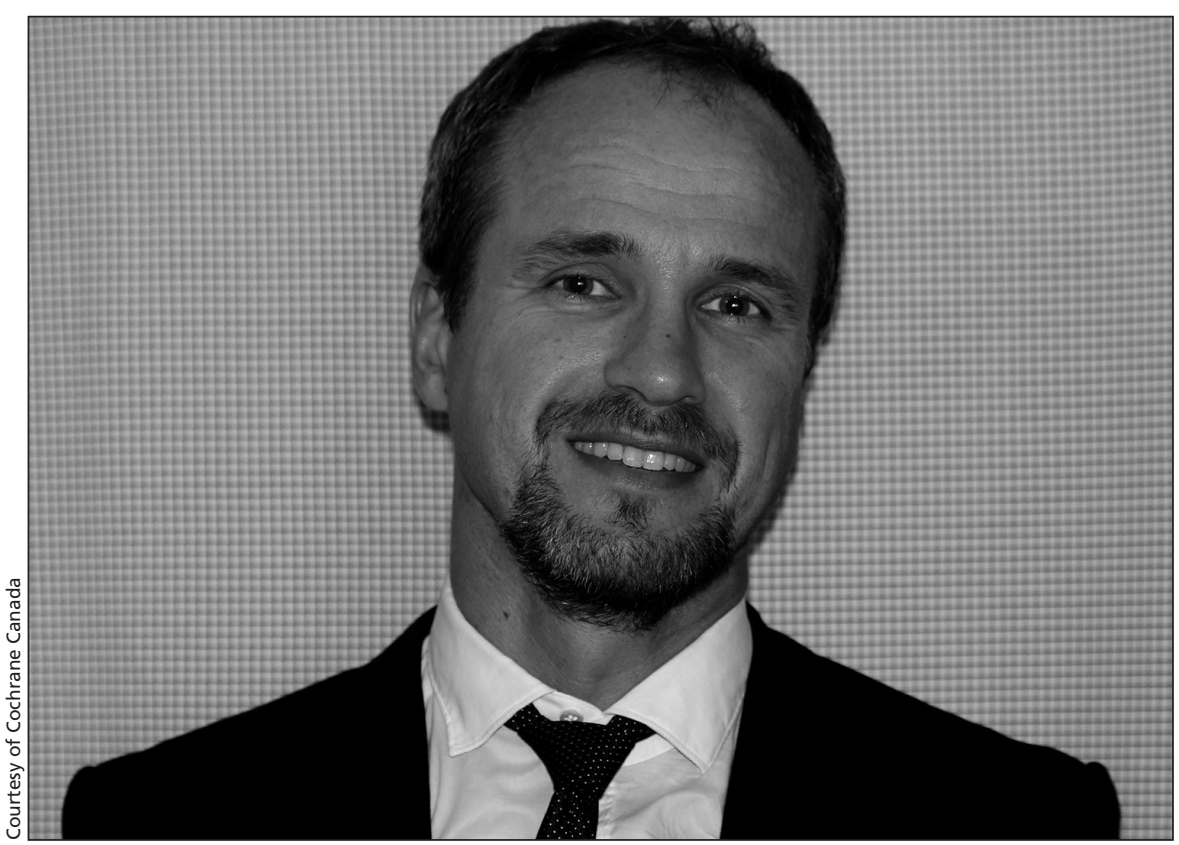

Cochrane Canada needs more secure funding, said its new director, Dr. Holger Schünemann.

A dual-funding model, with core peer-reviewed funding for Cochrane and open calls for other systematic reviews - an approach used in the United Kingdom and Australia, for example — could produce "huge synergies," he added.

Cochrane Canada has received bridge funding from McMaster University and Cochrane International, and the province of Ontario is considering a contribution, said Schünemann, who is chairman of the Department of Clinical Epidemiology \& Biostatistics at McMaster.

In addition to safeguarding its present operation, Cochrane Canada needs more secure funding to pursue new areas, such as bringing the basic sciences into the fold, working more closely with guideline developers and improving communication with clini- cians and other end-users to make the reviews more useful, said Schünemann.

Grimshaw, a senior scientist in the clinical epidemiology program at the Ottawa Hospital Research Institute said he intended to step down sooner, but remained because of the "funding crisis." "Given the commitment of the current government to evidence-informed decision-making in health, it is likely that there will be some solution in the short or medium term. I think wise heads will prevail."

Cochrane Canada recently relocated from the University of Ottawa to its original home at McMaster University in Hamilton, Ont. - Terry Murray, Toronto, Ont,

CMAJ 2016. DOI:10.1503/cmaj.109-5267 